\title{
Comment on "Nuclear localization of LDL receptor-related protein 1B in mammary gland carcinogenesis"
}

\author{
Chengyu Yang ${ }^{1} \cdot$ Long Zhao $^{1} \cdot$ Xuemei Liu $^{1} \cdot$ Ruixia Ma ${ }^{1} \cdot$ Yan Xu $^{1}$
}

Received: 17 February 2019 /Revised: 28 February 2019 / Accepted: 8 March 2019 / Published online: 15 March 2019

(C) Springer-Verlag GmbH Germany, part of Springer Nature 2019

To the editor:

We read with great interest the article by Asano and colleagues [1]. The study illuminated that the nuclear-localized LRP1B intracellular domain promoted breast cancer progression with poor prognosis, which possibly through the NEAT1 pathway. The results are very helpful to find new therapeutic target for breast cancer patients. On the other hand, from our perspective, the bioinformatics analyses need further context as the statistics for differential fold changes in expression data are not explained fully. For example, the authors seem to use unadjusted $p$ values for detecting differentially expressed genes (DEGs) regulated genes by nuclear-localized LRP1B intracellular domain. Due to the high false positives caused by a large number of probes and multiple comparisons, it seems essential to analyze microarray data properly to reach a reliable result by a statistical method. Only selecting DEGs with unadjusted $p$ values $<0.05$ in expression is not reliable and suitable for high-level microarray analysis.

Moreover, we would like to suggest using specialized highlevel microarray analysis such as LIMMA (linear models for microarray analysis) [2], commonly used for statistical testing and analysis of differential expression data by using linear models, and choosing more than 1.5 -fold expression changes and false discovery rate (FDR) $<0.05$ as the cutoff is an appropriate and conservative approach to obtain DEGs [3]. Moreover, modified significant analysis of microarray [4] is another and considerable non-parametric statistical algorithm; a 2-fold expression change and FDR $<0.1$ is a rational cutoff to obtain DEGs.

In addition, choosing the optimal statistical approach [5] and obtaining accurate and convincing results of DEGs analysis are basis for further data analysis such as gene network

Yan Xu

xuyanqyfy@126.com

1 Department of Nephrology, The Affiliated Hospital of Qingdao University, 16 Jiangsu Road, Qingdao 266003, China analysis. We welcome the authors to offer further explanation of their data analysis and experimental approach. We suggest transcriptomics data-intensive research would benefit from these considerations and innovations in statistical and data analytical approaches.

\section{References}

1. Asano Y, Takeuchi T, Okubo H, Saigo C, Kito Y, Iwata Y, Futamura M, Yoshida K (2019) Nuclear localization of LDL receptor-related protein $1 \mathrm{~B}$ in mammary gland carcinogenesis. J Mol Med (Berl) 97(2):257-268

2. Law CW, Alhamdoosh M, Su S, Smyth GK, Ritchie ME (2016) RNA-seq analysis is easy as 1-2-3 with limma, Glimma and edgeR. F1000Research 5:1408

3. Solano-Aguilar G, Molokin A, Botelho C, Fiorino AM, Vinyard B, Li R, Chen C, Urban J Jr, Dawson H, Andreyeva I, Haverkamp M, Hibberd PL (2016) Transcriptomic profile of whole blood cells from elderly subjects fed probiotic bacteria lactobacillus rhamnosus GG ATCC 53103 (LGG) in a phase I open label study. PLoS One 11(2): e0147426. https://doi.org/10.1371/journal.pone.0147426

4. Hossain MR, Bassel GW, Pritchard J, Sharma GP, Ford-Lloyd BV (2016) Trait specific expression profiling of salt stress responsive genes in diverse rice genotypes as determined by modified significance analysis of microarrays. Front Plant Sci 7:567. https://doi.org/ 10.3389/fpls.2016.00567

5. Chrominski K, Tkacz M (2015) Comparison of high-level microarray analysis methods in the context of result consistency. PLoS One 10(6):e0128845. https://doi.org/10.1371/journal.pone.0128845

Publisher's note Springer Nature remains neutral with regard to jurisdictional claims in published maps and institutional affiliations. 